\title{
A photoswitchable "host-guest" approach for the selective enrichment of dimethoate from olive oil
}

\author{
Raquel Garcia a, b, *, Elisabete P. Carreiro ${ }^{\text {c }}$, João P. Prates Ramalho ${ }^{\text {c, d }}$, Anthony J. Burke ${ }^{\text {c, d }}$, \\ João C. Lima ${ }^{\mathrm{b}}$, Marco D.R. Gomes da Silva ${ }^{\mathrm{b}}$, Ana Maria Costa Freitas ${ }^{\mathrm{e}}$, Maria João Cabrita ${ }^{\mathrm{e}}$ \\ a ICAAM - Instituto de Ciências Agrárias e Ambientais Mediterrânicas, IIFA, Universidade de Évora, Núcleo da Mitra, Ap. 94, 7006-554 Évora, Portugal \\ ${ }^{\mathrm{b}}$ LAQV, REQUIMTE, Departamento de Química, Faculdade de Ciências e Tecnologia, Universidade Nova de Lisboa, 2829-516 Caparica, Portugal \\ ${ }^{\text {c } C e n t r o ~ d e ~ Q u i ́ m i c a ~ d e ~ E ́ v o r a, ~ I I F A, ~ U n i v e r s i d a d e ~ d e ~ E ́ v o r a, ~ C o l e ́ g i o ~ L . A . ~ V e r n e y, ~} 7000$ Évora, Portugal \\ d Departamento de Química, Escola de Ciências e Tecnologia, Universidade de Évora, Colégio L.A. Verney, 7000 Évora, Portugal \\ e Departamento de Fitotecnia, Escola de Ciências e Tecnologia, ICAAM, Universidade de Évora, Pólo da Mitra, Ap. 94, 7006-554 Évora, Portugal
}

\section{H I G H L I G H T S}

\section{- Photocontrollable molecularly imprinted-based sorbent for selective recognition of dimethoate has been successfully prepared. \\ - Mechanisms underlying template recognition and uptake/release of the analyte have been explored by quantum chemical approach. \\ - Photoswitchable "host-guest" molecularly imprinted approach for dimethoate analysis in olive oil has been implemented.}

\section{A R T I C L E I N F O}

\section{Article history:}

Received 1 March 2018

Received in revised form

4 July 2018

Accepted 8 July 2018

Available online 11 July 2018

\section{Keywords:}

Food

Olive oil

Dimethoate

Sample preparation

Molecularly imprinted polymers

Photonic responsiveness
G R A P H I C A L A B S T R A C T

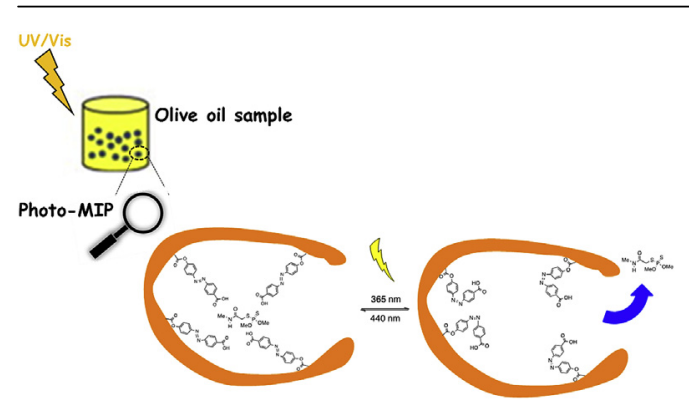

\begin{abstract}
A B S T R A C T
This work describes the development of a new selective photocontrollable molecularly imprinted-based sorbent for the selective enrichment/pre-concentration of dimethoate from spiked olive oil samples. To achieve this goal an improved molecularly imprinted strategy relying on the embedding of a functional monomer containing an azobenzene chromophore as light-responsive element, on the crosslinked tridimensional molecular imprinted network, has been assessed. To address the mechanisms underlying template recognition and uptake/release of the analyte from the functional imprinted material, computational studies using a quantum chemical approach, have been explored. This new functional sorbent provides a straightforward controllable uptake/release of the target template using light as the stimuli tool, which is highly advantageous due to light manipulation characteristics, such as superior clean, precision and remote controllable properties. In general, this work will contribute to the implementation of a photoswitchable analytical methodology that proves to be suitable for the selective isolation and further quantification of dimethoate from olive oil matrices at levels similar to the maximum residues limits imposed by the legislation. The limits of detection, calculated based on $3 \sigma$, was $1.6 \mathrm{mgL}^{-1}$ and the limit of quantification, based on $10 \sigma$, was $5.2 \mathrm{mgL}^{-1}$. The implemented sample preparation shows high reproducibility and recoveries $(93.3 \pm 0.4 \%)$.
\end{abstract}

() 2018 Elsevier B.V. All rights reserved.

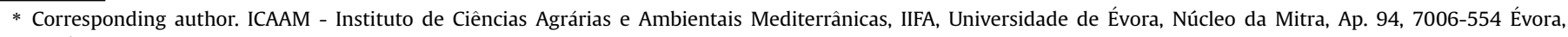
Portugal.
}

E-mail addresses: raquelg@uevora.pt,rm.garcia@fct.unl.pt (R. Garcia). 


\section{Introduction}

The presence of residual pesticides in food have gained increasing public concern and moreover in the last years has become one of the hot global food safety issues. Organophosphorus compounds are extensively applied in agriculture throughout the world to enhance agricultural productivity and improve the quality of agricultural products. In particular, organophosphorus compounds are widely applied in olive groves, thus increasing the likelihood of the presence of these residues in olive-derived foods. In fact, recent studies have shown that these residues (mainly insecticides and fungicides) can be found in olives and olive oil, mainly due to the lipophilic nature of these contaminants [1]. Aware of the harmful effect of pesticide residues, European Union and the Codex Alimentarius Committee on Pesticide Residues and the Agriculture Organization of the United States (FAO) have established maximum pesticide residue limits (MRLs) for olive oil [2]. The attainment of the desired low MRLs enforced by the legislation, has prompted the development of more sensitive and robust analytical techniques that enables an accurate determination of pesticide residue levels. Huge improvements have been performed on the development of more appropriate analytical methodologies for pesticide detection, mainly based on chromatographic techniques, like GC/MS and LC/MS, nevertheless these techniques are expensive and dependent of specialized laboratory staff [3]. Thus, the availability of a straightforward, reliable and sensitive methodology that enables the quantification of trace levels of pesticide residues in a very complex matrix-like olive oil, is highly desirable. Due to the considerable complexity of this food matrix, it is mandatory the integration of a sample preparation step in the analytical methodology, which provides a pre-concentration and/or enrichment of the analyte. It allows one to reduce the quantity of interferents in the background, improving the sensitivity of the analytical methodology. Sorbent materials are often used, in these pre-treatment procedures, they constitute the core of the sorption-based extraction techniques, playing a determinant role on the analyte retention process [4,5]. Consequently, the sorbent material will determine the selectivity and efficiency of the analytical methodology. However, commercially available sorbent materials, are limited and not effective for all types of analytes. In fact, non-specific sorbents (mostly employed as Solid Phase Extraction media) include surface-modified silicas and porous polymers (e.g., polystyrene-divinylbenzene resins and carbonbased materials). Despite, surface-modified silicas present a broader range of applications, some limitations could be ascribed, namely the limited stability when submitted to aqueous samples with low or high pH. Adsorptive inorganic oxides [e.g., silica, alumina and Florisil (magnesium silicate)] are being also used as sorbents, however their suitability is limited by the nature of such materials and of the analyte-adsorbent interactions [6]. Thus, the development of more selective sorbent materials for application on sample preparation purposes is a widespread hot research topic. Nowadays, the development of emerging functional nanomaterials for the detection of food contaminants constitute a hot research area [7].

Over the last years, the role of artificial "tailor-made" molecularly imprinted polymers (MIPs) as surrogate of natural receptors has prompted their application as selective sorbents on the sample preparation field. MIPs are synthetic materials mimicking natural receptors that possess artificially generated recognition sites able to rebind a target analyte even in the presence of structural analogues [8]. In fact, the use of a molecular imprinting strategy for the development of new sorbents opens a new way to apply the known "key-to-lock" biological model into the sample preparation field, enabling the interaction and the recognition of the analytes based on a specifically complementary framework that relies on a threedimensional polymeric network (MIPs). Therefore, MIP-based sorbents have proven to be valuable sample preparation tools for Solid Phase Extraction (SPE) purposes, known as MISPE [9]. Moreover, the merging of SPE with microscale extraction techniques [10], such as fiber/monolithic fiber solid-phase microextraction (SPME) and stir-bar sorptive extraction (SBSE) [11,12] has also been explored. Moreover, our research group have been focused on the development of MIP-based SPE sorbents for the isolation of several pesticide residues with relevance to olive oil production [13-17]. Recently, the isolation/pre-concentration of dimethoate from spiked olive oil samples has been implemented successfully using a MISPE-based methodology [13,18]. More recently, a new formatted MIP combining the pre-defined selectivity for dimethoate with a magnetic feature has proved to be a very promising smart-material for the development of selective sample preparation methodologies for the pre-concentration of this target pesticide in a very highly complex food matrix, as olive oil [17]. This procedure enables a straightforward separation of the analyte from the food matrix by means of magnetic separation, which is highly advantageous in the food sample preparation field.

The properties of MIPs, namely reusability, chemical and mechanical stability are in line with green chemistry concepts. However, the reagents and solvents used on their synthesis are hazardous and some environmental concerns are ascribed. Nevertheless, the rational design of MIPs with the view of addressing the environmental impact caused by the generation of unsuccessful imprints could overcome this drawback.

Nowadays, the exploitation of multi-functional-based sorbents possessing stimuli-regulated features for sample preparation is arousing huge interest. These stimuli-responsive materials, also known as smart materials, allow a switchable control of the uptake/ release of the analyte during the enrichment/pre-concentration step, constituting a significant improvement on the sorptionbased extraction techniques. More recently, a new approach that integrates stimuli-responsive elements into the MIP threedimensional polymer network has been explored, bringing together: i) the selectivity imprinted by the use of the molecular imprinting approach and ii) the switchable control of the uptake/ release processes by means of the incorporation of smart elements in the polymeric matrix [19]. Despite the huge potential that a switchable tool for sample preparation has on food science applications, the research developed until now in this area is still relatively unexplored [19]. Nowadays, photo irradiation is emerging as new external stimuli for stimuli-responsive polymeric materials $[20,21]$ since it is convenient to apply and easy to control. The development of molecularly imprinted polymers involves the incorporation of photosensitive chromophores, like azobenzene, stilbene or spiropyran, into the main chain of the polymer. The uptake/release of the target analyte is promoted by the UV-Vis photo-induced trans-cis isomerization of the chromophore owing to the photo-induced changes in orientation and space resulting in a significant alteration of receptor geometry, which affects the binding capacity of the host-guest imprinting system. These photo controllable sorbents could be potentially applied in several fields, namely separation/extraction, intelligent chemical carriers and catalysis [20,21].

Thus, the present work aims to enhance the knowledge and usefulness of photoswitchable tools for sample preparation applications by i) exploration of the design and development of a photo controllable molecularly imprinted polymer for the selective enrichment of dimethoate (Dmt) from olive oil matrices; ii) use of a computational quantum chemical approach to elucidate the mechanism of interconversion of azobenzene conformers by means of photoisomerization and moreover the mechanisms underlying 
template recognition on these photoresponsive polymeric systems. To achieve this main goal, a photoresponsive MIP integrating an azobenzene derivative (4-[(4-methacryloyloxy)phenylazo]benzoic acid (MPABA)) as a light-responsive element was synthesized and fully-characterized. This work comprises both experimental and theoretical studies of the photoisomerization process undergone by the light responsive element due to their crucial role on the uptake/ release of Dmt during the pre-concentration/isolation of the target analyte. Computational studies using a quantum chemical approach have also been performed to explore the mechanisms underlying template recognition and uptake/release of the analyte. Moreover, these studies also provide new insights into the role of the photoswitching core element during the pre-concentration/ isolation step of the sample preparation analytical workflow. Further, the new photoresponsive MIP has been successfully applied on the selective enrichment/pre-concentration of Dmt from olive oil spiked samples. The quantification of Dmt levels has been performed by HPLC/DAD. High reproducibility and recoveries $(93.3 \pm 0.4 \%)$ have been obtained proving the usefulness of the implemented photocontrollable MIP sorbent-based analytical workflow for the selective enrichment of Dmt from a highly complex food matrix, like olive oil.

Moreover, the present work represents a key innovation on the field of pesticide trace analysis in high complex food matrix since, to the best of our knowledge, is the first strategy for the development of a photo responsive material, to be applied as sorbent for solid phase extraction, that combines the concept of molecular imprinting with stimuli-responsive elements. It allows the implementation of a selective photoswitchable tool, that allows a straightforward way to control the uptake/release of the target pesticide-dimethoate, constituting a valuable food sample preparation methodology. Furthermore, the synthesis of Photo-MIPs could be tuned to other contaminants with relevance for the field of food safety and this methodology could be extended to other applications.

\section{Materials and methods}

\subsection{Chemicals}

1,1'-Azobisisobutyronitrile (AIBN) (Aldrich), trimethylolpropane trimethacrylate (TRIM) (Aldrich), $p$-aminobenzoic acid (Acros), $\mathrm{NaNO}_{2}$ (Riedel - deHaën), phenol (Riedel - deHaën), Hydrochloric acid ( $\mathrm{HCl}$ ) 37\% (Chem-Lab), NaOH (José Manuel Gomes dos Santos), Acetonitrile (Carlo Erba) were used without further purification. DMF and DMSO were distilled under reduced pressure and stored over $4 \AA$ molecular sieves. 4-[(4-Methacryloyloxy)phenylazo]benzoic acid (MPABA) was synthesized according to the literature method [22-24] (presented in Supplementary Materials Scheme S1). The water used in all experiments was distilled and purified by a Milli-Q system (Millipore, Bedford, MA, USA). HPLC grade acetonitrile and methanol, $n$-heptane were purchased from VWR International S.A.S. (Fontenay - Sons-Bois, France). The analytical standard Dmt was purchased from Sigma-Aldrich (Bellefonte, PA, USA) and was used without further purification. Prior to HPLC injection, all samples were filtered through $13 \mathrm{~mm}$ syringe filters ( $\mathrm{w} /$ $0.45 \mu \mathrm{m}$ PTFE membrane) (VWR, USA). The organic extra virgin olive oil was purchased from a local supermarket.

\subsection{Instrumentation}

${ }^{1} \mathrm{H}$ NMR spectra were recorded on a Bruker Avance III at $400 \mathrm{MHz}$. FTIR spectroscopy analysis measurements (in the range $450-4000 \mathrm{~cm}^{-1}$ ) were performed on a PerkinElmer Spectrum Two IR spectrophotometer. For the photoregulated uptake and release studies, a Hamamatsu L9588-06 Spot Light Source UV-lamp was used coupled to a monochromator (Jobin Yvon, Horiba, H10 UV model). All the chromatographic measurements were performed using a HPLC Waters Alliance 2695-series Separation Module equipped with Alliance Series Column Heater and the detection was carried out using a photodiode array detector (2998 PDA Detector) (Waters, USA). Chromatographic experiments were carried out with a LiChroCART C18 Purospher STAR reverse phase column $(250 \times 4.6 \mathrm{~mm}$ ID, $5 \mu \mathrm{m})$ (Merck Millipore, Germany) and the detection has been performed in the range of $190-600 \mathrm{~nm}$. Empower 3 FR2 software was used for management, acquisition and treatment of data. Chromatographic conditions used in each assay were described in detail in sections 2.2.2 and 2.4.

\subsection{Synthesis of Photo-MIP and Photo-NIP}

Synthesis of Photo-MIP and Photo-NIP: (4:1:24) MPABA (0.155 g, $0.5 \mathrm{mmol}$ ) and Dmt (28.6 mg, $0.125 \mathrm{mmol}$ ) were added to a $50 \mathrm{~mL}$ flask, and dissolved in DMF ( $3 \mathrm{~mL})$. TRIM $(0.96 \mathrm{~mL}, 3.0 \mathrm{mmol})$ was then added and the mixture was ultrasonicated at $0{ }^{\circ} \mathrm{C}$ for $30 \mathrm{~min}$ under a nitrogen atmosphere in the dark. Acetonitrile $(5 \mathrm{~mL})$ was added to the mixture that was then placed in a $70^{\circ} \mathrm{C}$ oil bath until all reagents were satisfactorily solubilized, AIBN ( $150 \mathrm{mg}$ ) was then added. However, to improve the solubility of the reagents a further quantity of DMF $(3 \mathrm{~mL})$ and DMSO $(1 \mathrm{~mL})$ were added. The polymerization occurred over $18 \mathrm{~h}$ at $70^{\circ} \mathrm{C}$ in the dark. The monolithic polymer obtained was filtered and the Dmt was removed by Soxhlet extraction with methanol/acetic acid $(9: 1, v / v)$ over a period of $48 \mathrm{~h}$ followed by methanol for a further $24 \mathrm{~h}$ in the dark. The resultant MIP material was dried to constant weight under vacuum at $60^{\circ} \mathrm{C}$. The Photo-NIP was also synthesized using the same method but in the absence of Dmt. Both materials were stored at room temperature in the dark. The Photo-MIP and Photo-NIP obtained were characterized using FTIR spectroscopy.

\subsection{Photoregulated uptake and release studies}

\subsubsection{Photoisomerization assays of MPABA}

To address the photoisomerization properties of MPABA, the following procedure has been applied: MPABA $(5 \mathrm{mg})$ was dissolved in 2-propanol $(3.5 \mathrm{~mL})$ and the corresponding UV-Vis spectra was obtained (named as initial time $\left(t_{0}\right)$ ). The sample in a quartz cuvette spectrometer cell and protected from exposure to ambient light, was irradiated at $365 \mathrm{~nm}$, using the combination of a Spot Light Source UV-lamp and a monochromator. The UV-Vis spectra of the solution were measured every $30 \mathrm{~min}$, for $90 \mathrm{~min}$. The procedure was repeated by irradiating the sample at $440 \mathrm{~nm}$. During the irradiation process, the samples were stirred continuously using a stirrer to assure the homogenization of the solution.

\subsubsection{Photoswitchable studies and evaluation of the molecular recognition of Photo-MIP}

Photoregulated uptake and release of Dmt by the Photo-MIP were assessed by means of switching on/off the UV light irradiation of the mixtures of Photo-MIP and analytes. From these assays, the molecular recognition of the Photo-MIP was also evaluated: $50 \mathrm{mg}$ of Photo-MIP was added to a quartz spectrometer cuvette containing $1.5 \mathrm{~mL}$ of a standard solution of Dmt in $n$-heptane (the final Dmt mass was $2.0 \mu \mathrm{g}$ ). The resultant mixture was irradiated at $440 \mathrm{~nm}$ for $90 \mathrm{~min}$. Then, the supernatant was separated, evaporated to dryness, reconstituted with $1 \mathrm{~mL}$ of acetonitrile and analyzed by HPLC/DAD. Methanol $(2 \mathrm{~mL})$ was added to the spectrometer cell containing the Photo-MIP, followed by irradiation at $365 \mathrm{~nm}$ during $90 \mathrm{~min}$. The liquid fraction was separated, evaporated to dryness, reconstituted with $1 \mathrm{~mL}$ of acetonitrile and 
analyzed by means of HPLC/DAD. The chromatographic conditions used were: the binary mobile phase consisted of solvents $A$ (water) and B (acetonitrile) with the following gradient: $25-100 \%$ B from 0 to $7 \mathrm{~min}$, then $100 \%$ B from 7 to $14 \mathrm{~min}$, after that $100-25 \%$ B from 14 to $19 \mathrm{~min}$, followed by $25 \% \mathrm{~B}$ from 19 to $24 \mathrm{~min}$. The elution flow rate was set at $0.5 \mathrm{~mL} \mathrm{~min}{ }^{-1}$ during the entire chromatographic process. The injection volume was $25 \mu \mathrm{L}$; the column temperature was set at $25^{\circ} \mathrm{C}$; DAD detection was performed at $220 \mathrm{~nm}$ and the detection to monitor the UV-Vis spectra was set between 190 and $600 \mathrm{~nm}$. All the experiments were conducted in triplicate and the average value taken. In order to access the degree of non-specific binding, a similar procedure was adopted for the corresponding Photo-NIP.

In order to guaranty that the batch of Photo-MIP prepared shows the same molecular recognition behavior, a complementary assay has been performed encompassing the repetition of the assay describe above with three different $50 \mathrm{mg}$ portions of Photo-MIP.

\subsection{Molecular modelling of photo-MIP-Dmt}

Structure optimizations were performed at DFT level, with the B97-D functional [25] as implemented in the GAMESS-US program [26]. Contrary to most exchange-correlation functionals that don't consider dispersion interactions, the B97-D functional designed by Grimme based on the pure GGA Becke 97 functional, contains an empirical correction to include dispersion interactions. The standard $6-31 G(d)$ basis set was employed for all atoms. Single point energies where calculated with the larger triple- $\zeta$ basis set 6 $311 G(d, p)$. TDDFT was used for calculating the low-lying excited states and electronic transitions of the monomers, by using the hybrid CAM-B3LYP functional [27] together with the $6-311+G(d, p)$ basis set. The equations were solved for 40 excited states. To take into account the solvent effects, the SMD continuum solvation model method [28] was employed.

\subsection{Implementation of the analytical methodology for the selective extraction of Dmt in spiked olive oil samples}

The organic extra virgin olive oil used in this work was free of Dmt (confirmed by HPLC/DAD analysis), and the spiking concentration was $2.0 \mu \mathrm{g} \mathrm{g}^{-1}$, which correspond to the MRLs for this pesticide in olive products [2]. The experimental procedure is outlined in Fig. 1.

Since olive oil is a very complex matrix, an improved version of the chromatographic method described in Section 2.2 has been used to ensure an efficient discrimination of the peak corresponding to the target analyte (Dmt) avoiding its eventual coelution with some matrix interferents. The chromatographic conditions used to perform these studies were the following: a binary mobile phase consisted of solvents $A$ (water) and $B$ (acetonitrile) as follows: $25-100 \%$ B from 0 to $80 \mathrm{~min}$, then $100 \%$ B from 80 to $85 \mathrm{~min}$, followed by $100-25 \%$ B from 85 to $90 \mathrm{~min}$ and, after that $25 \%$ B until $95 \mathrm{~min}$; the flow rate was fixed at $0.4 \mathrm{~mL} \mathrm{~min}^{-1}$ during the entire chromatographic process. The injection volume was $25 \mu \mathrm{L}$; a temperature of $25^{\circ} \mathrm{C}$; DAD detection was performed at $220 \mathrm{~nm}$. All the determinations were conducted in triplicate and the average value calculated.

\subsection{Experimental validation (calibration curves/repeatability)}

The identification of the target pesticide (Dmt) was achieved by comparison of its retention time and UV-Vis spectra with those of the corresponding standard. The quantification was determined by calculating the areas of the relevant chromatographic peaks obtained by UV detection at $220 \mathrm{~nm}$ using standard solutions of the pesticide with known concentrations. All experiments were conducted in triplicate, and the average value taken. The calibration curve of the Dmt $\left(y=3 \times 10^{7} x-5615.9\right)$ showed a linear range between 1 and $100 \mathrm{mg} \mathrm{L}^{-1}$, a LOD and LOQ of 1.6 and $5.2 \mathrm{mg} \mathrm{L}^{-1}$, respectively (calculated as 3 and 10 times the standard deviation $(\sigma))$ and a correlation coefficient $(r)$ of 0.9998 .

\section{Results and discussion}

\subsection{Synthesis of the Photo-MIP and the Photo-NIP}

4-[(4-Methacryloyloxy)phenylazo]benzoic acid (MPABA) was chosen as a photoresponsive functional monomer for the preparation of the Photo-MIP and the corresponding Photo-NIP, due to the affinity of its binding group-the carboxylic acid, for the target template (Dmt). The putative hydrogen bond interactions between MPABA and Dmt (during the prepolymerization step) that are crucial for the formation of MIP's binding cavity are depicted in Fig. 2. The MPABA functional monomer and its precursor, 4-[(4'hydroxy)phenylazo]benzoic acid, were synthesized using literature methods [22-24]. Their structures were confirmed by ${ }^{1} \mathrm{H}$ NMR spectroscopy (see Supplementary Materials, Fig. S1 and Fig. S2).

The Photo-MIP was synthesized using traditional bulk radical polymerization with a thermal free radical initiator, as represented in Fig. 2. To avoid photoisomerization of the azobenzene derivative chromophore, the synthetic procedure was carried out in the dark. Since MPABA is quite insoluble in acetonitrile and in other common solvents used for the MIP synthesis, the use of a mixture of dimethylformamide (DMF) and dimethylsulfoxide (DMSO) proved to be the most satisfactory to completely solubilize the functional monomer. So, a mixture of acetonitrile/DMF/DMSO (5:6:1, v/v/v) was selected as porogen. The crosslinker and the radical initiator used were TRIM and AIBN, respectively. In the synthetic procedure, a molar ratio of 1:4:24 for Dmt/MPABA/crosslinker was used. Moreover, a final template extraction removal was accomplished through Soxhlet extraction using a mixture of methanol/acetic acid $(9: 1, v / v)$ during $48 \mathrm{~h}$, followed by a repeated Soxhlet extraction using only methanol as solvent for $24 \mathrm{~h}$, affording the free imprinted cavities for the selective rebinding of the target pesticide. Imprinting systems were characterized by FTIR spectroscopy.

\subsection{Characterization of the photo-imprinted systems}

\subsubsection{FTIR}

The FTIR spectra are consistent with data reported in the literature [24]. Photo-MIP and Photo-NIP FTIR spectra are shown in the Supplementary Materials section (Fig. S3). As expected, it was possible to identify the typical bands in the spectra of the PhotoMIP according to the chemical structure of the imprinted systems. Thus, the band at ca. $3490 \mathrm{~cm}^{-1}$ can be attributed to stretching vibrations of the $\mathrm{O}-\mathrm{H}$ bond of MPABA. Also, the characteristic peaks at $1621 \mathrm{~cm}^{-1}$ and $1476 \mathrm{~cm}^{-1}$ can be attributed to a $\mathrm{C}=\mathrm{C}$ stretching band, and the band at $1400 \mathrm{~cm}^{-1}$ attributed to a $\mathrm{C}-\mathrm{N}$ bond stretching vibration. The band for the $\mathrm{C}-\mathrm{H}$ stretch appeared at $2999 \mathrm{~cm}^{-1}$. The presence of bands at $1157 \mathrm{~cm}^{-1}$ and $1270 \mathrm{~cm}^{-1}$ were attributed to a $\mathrm{C}-\mathrm{O}$ stretch, whilst the band at $1740 \mathrm{~cm}^{-1}$ can be attributed to a $\mathrm{C}=\mathrm{O}$ stretching vibration. As expected, the FTIR spectra for both the Photo-MIP and Photo-NIP are very similar.

\subsection{Photoregulated uptake and release studies}

\subsubsection{Photoisomerization assays of MPABA}

The photoisomerization process undergone by the light responsive monomer MPABA plays a key role on the design of the Photo-MIP and their further application as light-responsive 


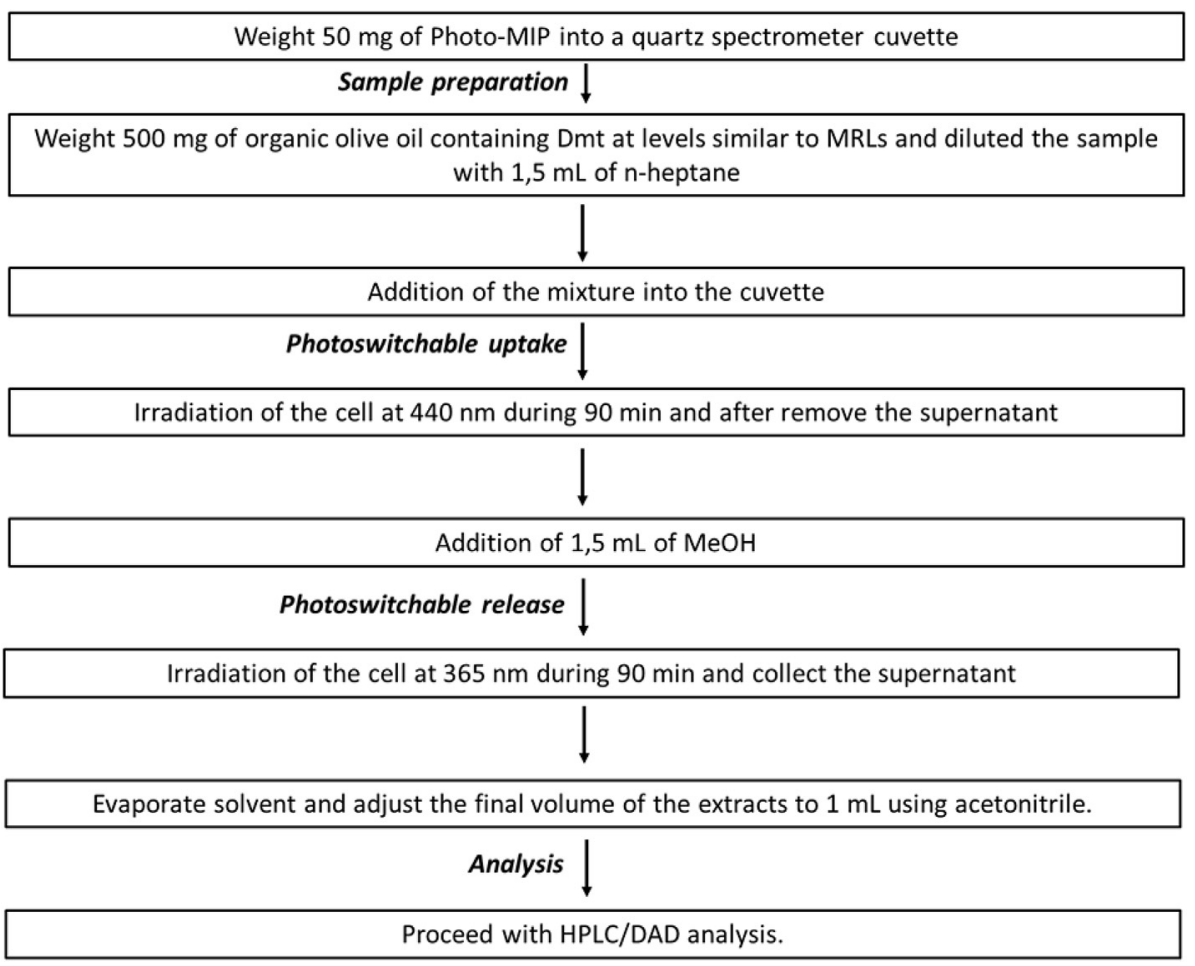

Fig. 1. Analytical workflow for the trace analysis of the target pesticide Dmt from spiked organic olive oil samples based on the use of Photo-MIP as sorbent.

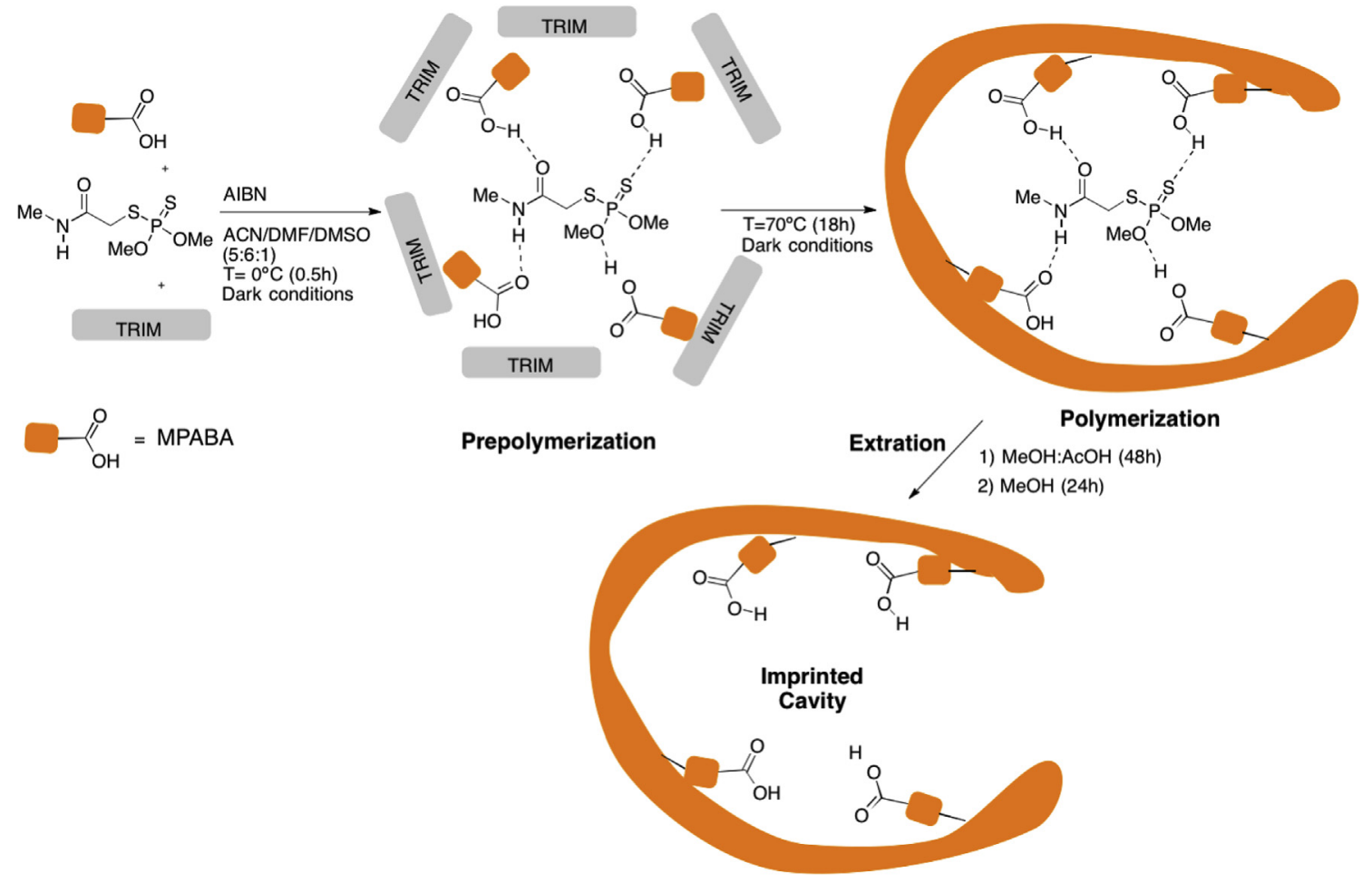

Fig. 2. Schematic representation of Photo-MIP synthesis.

sorbent-based on the development of the sample preparation methodology encompassed in this work.

The functional monomer MPABA can exist in either the cis- or trans-conformations, as represented in Fig. 3. As is the case for azobenzene, the trans MPABA isomer is planar and energetically more stable than the cis isomer, with a calculated energy gap of $9.5 \mathrm{kcal} \mathrm{mol}^{-1}$ between the two isomers. The occurrence of these two conformations is crucial for the photocontrollable process that underlines the desired template recognition of the Photo-MIP enabling an autoregulated uptake/release process. Prior to 

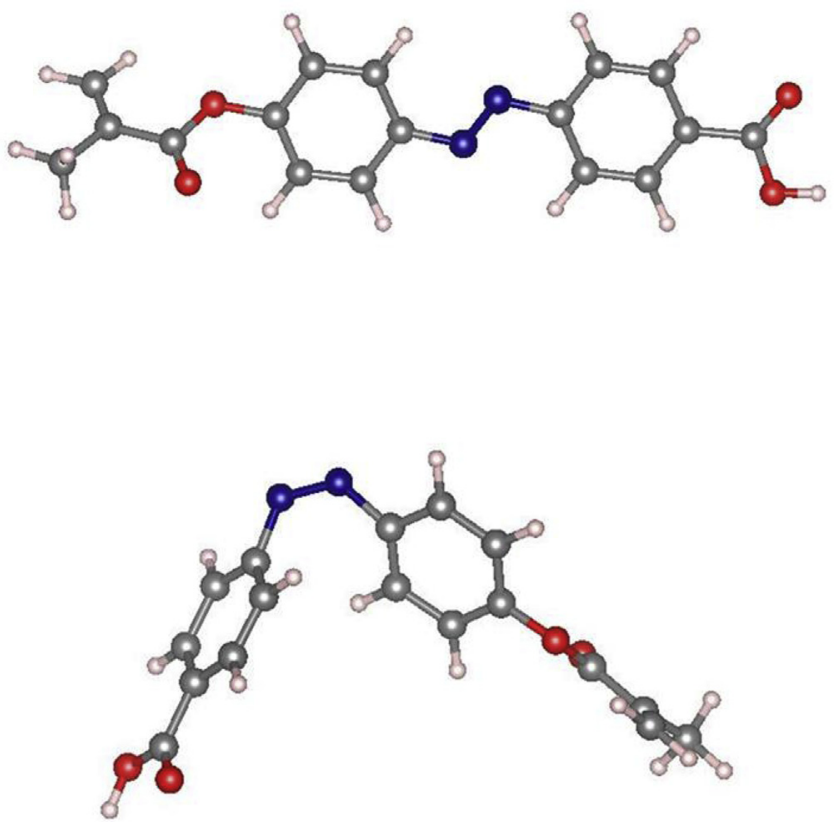

Fig. 3. Optimized molecular structures at DFT level of the MPABA trans (up) and cis (bottom).

conducting these experiments, it was essential that a photoisomerization study of the functional monomer (MPABA), which is used for the synthesis of the Photo-MIP, was performed due to its key role in the photoregulation of the resulting imprinted system. The relevance of MPABA for the development of these kind of imprinting systems is due to the presence of a photoresponsive azobenzene chromophore in its structure, as well as the benzoic acid functionality for template interaction (via H-bonding) and a polymerizable metacryloyl group. Fig. 4 shows the simulated UVVis absorption spectra of the trans and cis conformers.

As shown in Fig. 4, trans-MPABA possesses one strong absorption band around $356 \mathrm{~nm}$ while cis- MPBA absorbs at $299 \mathrm{~nm}$. Also, a low lying band, around $473 \mathrm{~nm}$, is visible for the cis conformer while the corresponding transition at $468 \mathrm{~nm}$ is forbidden in the

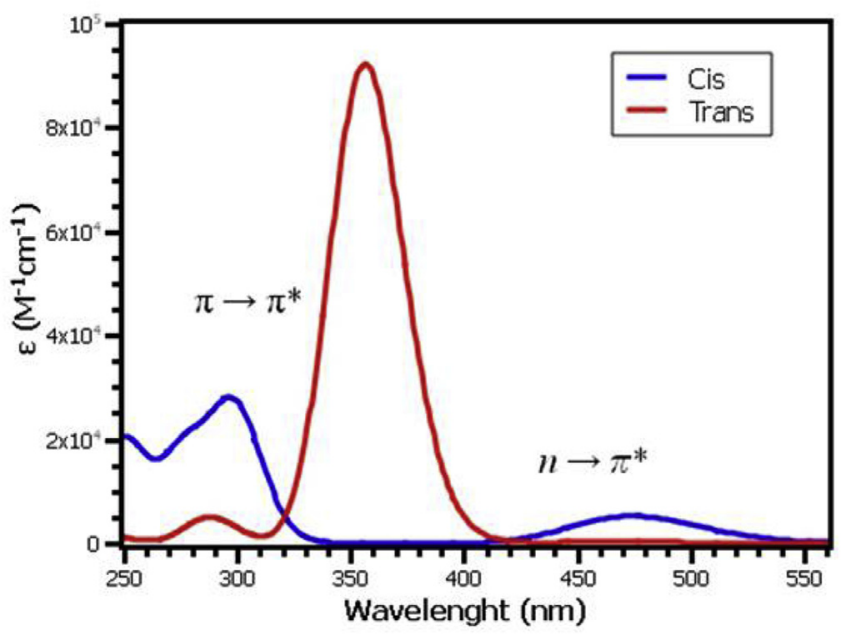

Fig. 4. Simulated UV-Vis absorption spectra of the trans and cis conformers of functional monomer MPABA. case of the of the trans derivative. This excitation becomes allowed for the cis conformer due to its nonplanar geometry. These two set of transitions are typical for the azo compounds and can be ascribed to $\pi \rightarrow \pi^{*}$ and $\mathrm{n} \rightarrow \pi^{*}$ electron transitions, respectively. Although the excitation energies are underestimated by $\sim 30-40 \mathrm{~nm}$, these results corroborate the data obtained in the experimental assays (as described below).

Experimentally, photoisomerization assays for MPABA have been performed and the obtained results are shown in Fig. 5. Upon irradiation at $365 \mathrm{~nm}$, the intensity of the $\pi-\pi^{*}$ transition decreased rapidly, while that of the $n-\pi^{*}$ transition showed a slight increase, occurring the trans-cis isomerization of the azobenzene chromophore. Otherwise, the irradiation of the cis-MPABA conformer using visible light $(440 \mathrm{~nm}$ ) leads to an increase of the intensity of the $\pi$ $\pi^{*}$ transition, as depicted in Fig. 5. Then, the data indicated that the photoinduced isomerization is reversible.

\subsubsection{Photoregulated studies of photo-MIP-Dmt}

As detailed above, the incorporation of MPABA as functional monomer on the crosslinked polymeric structure of the Photo-MIP enables a straightforward photoregulated isomerization of MPABA owing to the photoresponsive character of the azobenzene chromophore. The irradiation wavelengths of 365 and $440 \mathrm{~nm}$ were selected according to experimental UV-Vis absorption spectra and photoresponsive data. The selection of those wavelength values was also corroborated by the simulated UV-Vis absorption spectra of the trans and cis conformers of the functional monomer MPABA (as depicted in Fig. 4). This process constitutes a key step for the uptake/release of the target pesticide.

\subsection{Evaluation of the molecular recognition of Photo-MIP}

The molecular recognition of Photo-MIP has been assessed by means of a binding assay performed with a standard solution of Dmt at levels similar to the MRL imposed by legislation. The PhotoMIP showed a retention capacity of $85.3 \pm 0.8 \%$ and a corresponding value of $0.034 \mu \mathrm{gmg}^{-1}$ for the Photo-MIP capacity, while the corresponding Photo-NIP only presented a value of $62.1 \pm 0.3 \%$ for the retention capacity. Thus, this finding supports the suitability of the photo controllable sorbent for the selective enrichment/preconcentration of the target pesticide (Dmt). In terms of reproducibility of the molecular recognition behaviour of the Photo-MIP batch, the assays performed shows that the retention capacity varies in the range $85.6-86 \%$, using in the assays three different $50 \mathrm{mg}$ portions of the Photo-MIP (as detailed in the experimental section 2.2.2.).

Apart the photo-controllable nature of Photo-MIP, one of the most promising feature of this imprinting systems is the improvement on the extraction selectivity, comparatively with the common SPE-based sorbents. However, MIPs can also extract compounds structurally-analogous to the template molecule, a phenomenon designated as "cross-selectivity". Since this topic is of crucial relevance, this work has also encompassed a selectivity assay with omethoate-a dimethoate analogue, which presents a highly structural similarity with the pesticide under study. The recovery rate obtained using Photo-MIP as sorbent and omethoate as target template was $68 \pm 0.3 \%$, using concentrations similar to the MRL for the dimethoate and a similar procedure to that describe in section 2.2.2. This finding proves the suitability of Photo-MIP for the selective enrichment of dimethoate.

\subsection{Molecular modelling studies of Photo-MIP}

To gain insight on the interaction between the template and the Photo-MIP and, also on the uptake and release process of the 

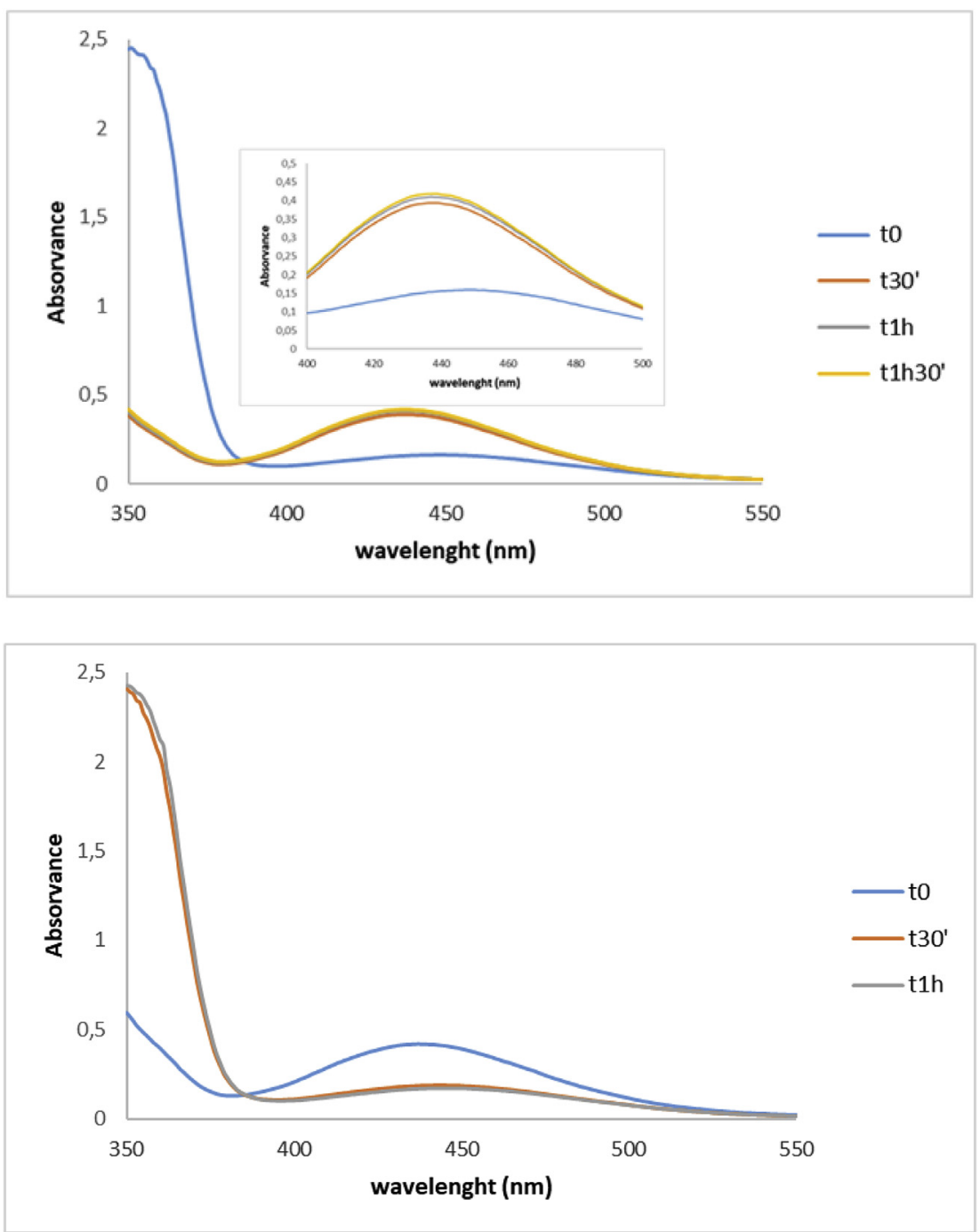

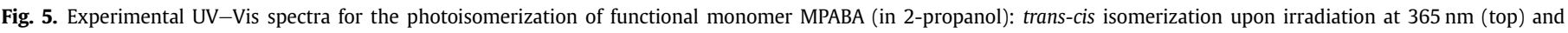
subsequent cis-trans isomerization by visible light irradiation at $440 \mathrm{~nm}$ (bottom).

template induced by the photoregulated procedure, a complex consisting of one template and four monomers was considered. The monomers were initially located at diverse sites on the template selected as the most likely to form the strongest bonds including the formation of hydrogen bonds. In this conformation the template carbonyl and the methoxy oxygens can act as hydrogen acceptors while the amide group can interact with a monomer as a hydrogen donor. All the monomers were in the trans conformations. The template-monomer complex was then fully optimized at the B97-D/6-31G(d) level. In a further step, one of the monomers was altered to the cis conformation and an additional restricted energy minimization was performed. In this second minimization the monomers ethenylmethyl moiety were kept in their previously optimized positions, restricting the monomers geometries, in order to mimetize the imprinted structure of the MIP acquired on the polymerization step. The template however was left totally free on this second energy minimization stage. The optimized geometries of the template-monomer complexes are depicted in Fig. 6.

The interaction energies $\Delta \boldsymbol{E}_{\mathbf{I}}$ in solution of the template with the monomer assemblies were calculated at the B97-D/6-311G $(\mathrm{d}, \mathrm{p})$ level as
$\Delta \boldsymbol{E}_{\mathbf{I}}=\boldsymbol{E}_{\text {Complex }}-\boldsymbol{E}_{\text {Template }}-\boldsymbol{E}_{(\text {Monomer })_{n}}$

with the template and monomer species kept in the complexes geometries. The solvents chosen were $n$-heptane and methanol, the solvents that have been used on enrichment/pre-concentration and elution steps of the template molecule from the polymeric structure of Photo-MIP, respectively.

The monomers and the template geometries were largely unaffected by the conformational change induced in one of the monomers. Although one hydrogen bond was broken upon the trans to cis modification of one of the monomers, the remaining hydrogen bonds kept the template almost in an unchanged geometry. Contrary to what takes place on the geometry, the energetic effects are significant after one monomer be converted to the cis form. With regards to the interaction of the template with the monomers, the results in Table 1 show that two effects are present. For both geometries, the interactions are stronger in heptane than in methanol. This is consistent with what was observed experimentally with heptane promoting a better binding capacity of the MIP towards the template than methanol. The weakest interaction 


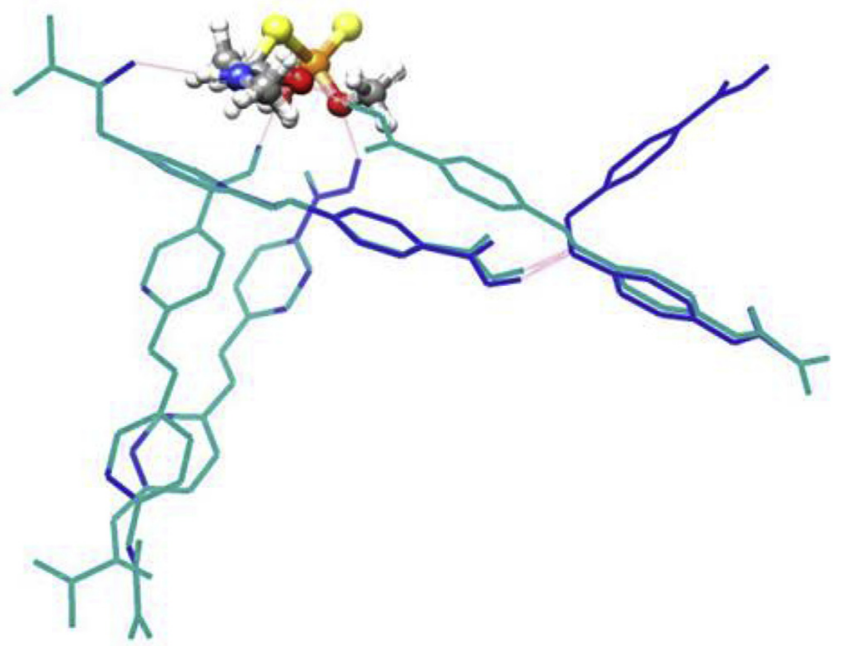

Fig. 6. Superimposed optimized structures of the template-(monomer) $)_{4}$ complex with the four monomers in the trans conformation (light blue) and with three monomers in trans and one in the cis conformation (dark blue). (For interpretation of the references to colour in this figure legend, the reader is referred to the Web version of this article.)

Table 1

Interaction energies $\left(\mathrm{kcal} \mathrm{mol}^{-1}\right)$ for the template-(monomer) $)_{4}$ system for the all trans monomers and after one monomer be converted in the cis form.

\begin{tabular}{lll}
\hline & \multicolumn{2}{l}{ Interaction energy } \\
\cline { 2 - 3 } & all-trans & 3-trans/1-cis \\
\hline$n$-heptane & -41.5 & -29.4 \\
Methanol & -33.8 & -24.2 \\
\hline
\end{tabular}

energies observed in methanol also makes it the preferred eluting solvent. Also, as expected, the conformational change of the monomer from trans to cis, reduces significantly the interaction energy of the monomers with the template as a reduction of the number of hydrogen bonds occurs when the trans to cis change takes place. This effect would be increased if more monomers suffer the trans to cis conformational change.

\subsection{Implementation of the analytical methodology for the selective} extraction of Dmt from spiked olive oil samples

Aiming to achieve the main goal of the work, this photoswitchable sample preparation tool has been validated for the selective extraction of Dmt from spiked olive oil samples, at levels similar to the MRLs enforced by the legislation (as outlined in Fig. 1). The sample preparation methodology has been successfully implemented since a high recovery rate of $93.3 \pm 0.4 \%$ has been achieved with a good precision ( $\mathrm{RSD}=0.43 \%$ ). The results obtained proves the suitability of the functional Photo-MIP as selective light responsive sorbent for the cleanup of the target pesticide in a very complex matrix-olive oil, even at levels corresponding to the MRL. Fig. 7 depicts the HPLC-DAD chromatograms of an extract of spiked olive oil sample using Photo-MIP as sorbent.

Recently, Bakas and co-workers have developed an MISPE-based methodology for the extraction of Dmt from olive oil, with recovery rates up to $94 \%$ [18]. Previously, our research group has also introduced a MIP selective for Dmt using an iniferter-synthetic molecular imprinting strategy. The new MIP has been used as SPE sorbent for the enrichment of Dmt from olive oil spiked samples [13]. However, both studies involved the packaging of the imprinted materials in an SPE syringe in order to perform the MISPE extractions. This procedure is laborious and time consuming. More recently, our research group have developed a magnetic molecularly imprinted - based analytical tool for the selective enrichment of dimethoate from olive oil samples. This approach combines magnetic separation and molecular imprinting features, in such a way that provides simplicity and versatility to the sample preparation methodology [17].

Thus, the present work overcomes some of the limitations ascribed to MISPE, namely: i) MISPE is often used in cartridge mode, which usually involves a tedious column-packing procedure; ii) template release is performed by using a large volume of solvent; and iii) MISPE is only applicable for extraction and concentration of small amounts of sample owing to the restricted amount of MIP packaging on the SPE column.

Since the reusability is a key factor on the implementation of sorption-based extraction techniques, this parameter has been evaluated using samples of spiked olive oils. The data obtained shows that the Photo-imprinted material can be used for the selective enrichment of Dmt for at least 20 utilizations without losses of recognition capacity. Indeed, in twentieth utilization, a recovery rate of $93.0 \%$ has been obtained, proving the lower carryover of Dmt by the reuse of PMIP.

Therefore, a photoswitchable sample preparation tool for the selective and remote controllable uptake/release of the target template using light as the stimuli-responsive element, without impairment of the selectivity, has been successfully implemented on the trace analysis of Dmt from olive oil spiked samples.

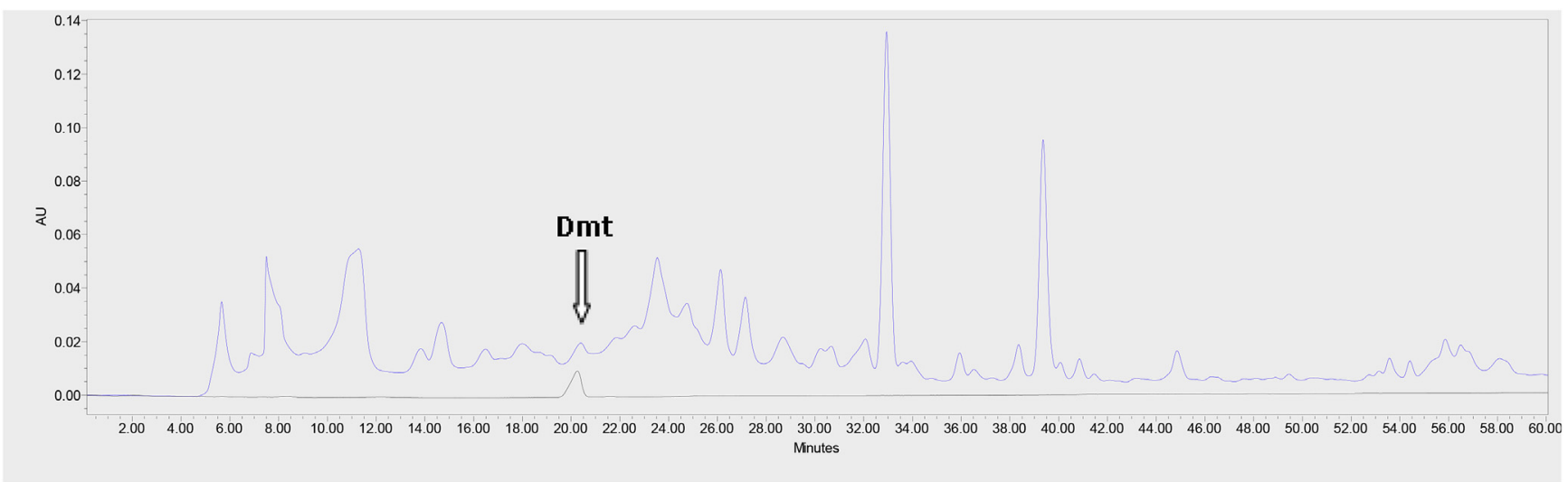

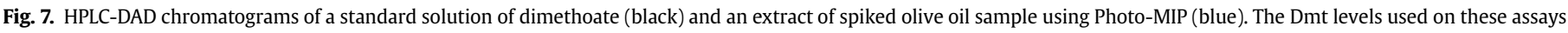
correspond to the MRL imposed by legislation. (For interpretation of the references to colour in this figure legend, the reader is referred to the Web version of this article.) 


\section{Conclusions}

A successful sample preparation methodology based on a "hostguest" interaction between the recognition cavities of a photo responsive MIP and the template molecule, for the selective enrichment/pre-concentration of Dmt at levels similar to the MRLs, has been implemented in very complex food matrices, as olive oil. Particularly, the incorporation of an azobenzene moiety in the MPABA functional monomer, which undergone a cis-trans isomerization upon irradiation with visible light enable to control the uptake/release of the target pesticide from this photo-responsive material. Since the geometry of the receptor sites returned to the original state, it allows the reuse of the Photo-MIP for at least 20 cycles of enrichment/preconcentration without loss of the molecular recognition, with considerable economic and environmental advantages. Additionally, new insights about the role of the photoswitching core element, that constitute the new functional material, during the pre-concentration/isolation step has been achieved using a quantum chemical approach.

In conclusion, the new imprinted sorbent displays a remarkable molecular imprinting effect, retains the photoisomerization properties of the azobenzene chromophore showing additionally a response to the light as remote controllable tool. The results obtained shows the potential applicability of the photo-MIPs for simultaneous and highly efficient and selective preconcentration, separation and accurate quantification of dimethoate in spiked olive oil samples. Moreover, the proving versatility of this photoswitchable sample preparation tool could be extended into a wide range of applications.

\section{Conflicts of interest}

The authors declare no conflict of interest.

\section{Acknowledgments}

This work has been supported by FEDER and National founds, through the Programa Operacional Regional do Alentejo (InAlentejo) Operation ALENT-07-0262-FEDER-001871Laboratório de Biotecnologia Aplicada e Tecnologias Agro-Ambientais and FEDER Funds through the Operational Programme for Competitiveness Factors -COMPETE and National Funds through FCT - Foundation for Science and Technology under the Project UID/AGR/00115/2013. This work was also supported by the Associate Laboratory for Green Chemistry LAQV which is financed by national funds from FCT/MEC (UID/QUI/50006/2013) and co-financed by the ERDF under the PT2020 Partnership Agreement (POCI-01-0145-FEDER-007265). Raquel Garcia and Elisabete P. Carreiro acknowledge the Fundação para a Ciência e Tecnologia (FCT) for the post-doctoral research fellowships- SFRH/BPD/109912/2015 and SFRH/BPD/72182/2010, respectively. We are grateful also for funding from FCT via the Strategic Project UID/QUI/0619/2016. We are grateful to project LADECA (ALENT-07-0262-FEDER-001878) for financing the acquisition of our Bruker Avance III NMR spectrometer.

\section{Appendix A. Supplementary data}

Supplementary data related to this article can be found at https://doi.org/10.1016/j.aca.2018.07.017.

\section{References}

[1] A. Ruiz-Medina, E.J. Llorent-Martínez, Evaluation of the contamination by herbicides in olive groves, in: Mohammed Naguib Abd El-Ghany Hasaneen (Ed.), Herbicides - Properties, Synthesis and Control of Weeds, first ed.,
Publisher: InTech, 2012, ISBN 978-953-307-803-8, pp. 207-226.

[2] Regulation (EC) No. 396/2005 of the European Parliament and of the council of 23 February 2005 on maximum residue levels of pesticides in or on food and feed of plant and animal origin and amending Council Directive 91/414/EEC, and subsequent updates, http://ec.europa.eu/food/plant/protection/ pesticides/index_en.htm and Codex Alimentarius Committee on Pesticide Residues, Joint FAO/WHO Meeting on Pesticide. http://www. codexalimentarius.net/mrls/pestdes/jsp/pest_q-e.jsp.

[3] M.F. Zayats, S.M. Leschev, M.A. Zayats, A novel method for the determination of some pesticides in vegetable oils based on dissociation extraction followed by gas chromatography-mass spectrometry, Food Addit. Contam. 33 (8) (2016) 1337-1345, https://doi.org/10.1080/19440049.2016.1209575.

[4] J. Płotka-Wasylka, M. Marć, N. Szczepańska, J. Namieśnik, New polymeric materials for solid phase extraction, Crit. Rev. Anal. Chem. 47 (2017) 373-383. https://doi.org/10.1080/10408347.2017.1298987.

[5] F. Augusto, L.W. Hantao, N.G.S. Mogollón, S.C.G.N. Braga, New materials and trends in sorbents for solid-phase extraction, TrAC. Trends Anal. Chem. 43 (2013) 14-23. https://doi.org/10.1016/j.trac.2012.08.012.

[6] J.F. García-Reyes, C. Ferrer, M.J. Gómez- Ramos, A. Molina- Díaz, A.R. Fernandez- Alba, Determination of pesticide residues in olive oil and olives, Trends Anal. Chem. 26 (3) (2007) 239-251. https://doi.org/10.1016/j. trac.2007.01.004.

[7] J.M. Liu, Y. Hub, Y.K. Yange, H. Liu, G.Z. Fang, X. Lu, S. Wang, Emerging functional nanomaterials for the detection of food contaminants, Trends Food Sci. Technol. 71 (2018) 94-106. https://doi.org/10.1016/j.tifs.2017.11.005.

[8] R. Garcia, M.J. Cabrita, A.M. Costa Freitas, Application of molecularly imprinted polymers for the analysis of pesticide residues in food-a highly selective and innovative approach, Am. J. Anal. Chem. 2 (2011) 16-25, https://doi.org/ 10.4236/ajac.2011.228119.

[9] A. Martín-Esteban, Molecularly-imprinted polymers as a versatile, highly selective tool in sample preparation, Trends Anal. Chem. 45 (2013) 169-181. https://doi.org/10.1016/j.trac.2012.09.023.

[10] G. Li, K.H. Row, Recent applications of molecularly imprinted polymers (MIPs) on micro-extraction techniques, Separ. Purif. Rev. 47 (2018) 1-18. https://doi. org/10.1080/15422119.2017.1315823.

[11] Y. Hu, J. Pan, K. Zhang, H. Lian, G. Li, Novel applications of molecularlyimprinted polymers in sample preparation, Trends Anal. Chem. 43 (2013) 37-52. https://doi.org/10.1016/j.trac.2012.08.014.

[12] N. Razavi Sarafraz-Yazdi, Application of molecularly-imprinted polymers in solid-phase microextraction techniques, Trends Anal. Chem. 73 (2015) 81-90. https://doi.org/10.1016/j.trac.2015.05.004.

[13] N. Martins, E.P. Carreiro, M. Simões, M.J. Cabrita, A.J. Burke, R. Garcia, An emerging approach for the targeting analysis of dimethoate in olive oil: the role of molecularly imprinted polymers based on photo-iniferter induced "living" radical polymerization, React. Funct. Polym. 86 (2015) 37-46. https:// doi.org/10.1016/j.reactfunctpolym.2014.11.003.

[14] R. Garcia, N. Martins, E.P. Carreiro, M. Simões, M.M.L.R. Carrott, P.J.M. Carrott, A.J. Burke, M.J. Cabrita, Development of a selective sorbent for solid-phase extraction of terbuthylazine in olive oil samples: a molecular imprintingbased strategy, J. Sep. Sci. 38 (2015) 1204-1212, https://doi.org/10.1002/ jssc.201401263.

[15] N. Martins, E.P. Carreiro, A. Locati, J.P.P. Ramalho, M.J. Cabrita, A.J. Burke, R. Garcia, Design and development of molecularly imprinted polymers for the selective extraction of deltamethrin in olive oil: an integrated computational assisted approach, J. Chromatogr. A 1409 (2015) 1-10. https://doi.org/10. 1016/j.chroma.2015.07.025.

[16] R. Garcia, E.P. Carreiro, J. Nunes, M.D.R. Gomes da Silva, A.M. Costa Freitas, A.J. Burke, M.J. Cabrita, Dual layer solid phase extraction based on molecular imprinting technology: seeking a route to enhance selectivity for trace analysis of pesticides residues in olive oil, Electrophoresis 37 (2016) 1916-1922, https://doi.org/10.1002/elps.201600073.

[17] R. Garcia, E.P. Carreiro, J.P.P. Ramalho, J. Mirão, A.J. Burke, M.D.R. Gomes da Silva, A.M. Costa Freitas, M.J. Cabrita, A magnetic controllable tool for the selective enrichment of dimethoate from olive oil samples: a responsive molecular imprinting-based approach, Food Chem. 254 (2018) 309-316, https://doi.org/10.1016/j.foodchem.2018.02.003.

[18] I. Bakas, N.B. Oujji, E. Moczko, G. Istamboulie, S. Piletsky, E. Piletska, E. AitAddi, I. Ait-Ichou, T. Noguera, R. Rouillon, Computational and experimental investigation of molecular imprinted polymers for selective extraction of dimethoate and its metabolite omethoate from olive oil, J. Chromatogr. A 1274 (2013) 13-18, https://doi.org/10.1016/j.chroma.2012.11.061.

[19] R. Garcia, M.D.R. Gomes da Silva, M.J. Cabrita, On-off" switchable tool for food sample preparation: merging molecularly imprinting technology with stimuli-responsive blocks. Current status, challenges and highlighted applications, Talanta 176 (2018) 479-484. https://doi.org/10.1016/j.talanta.2017. 07.082 .

[20] Y.-b. Wei, Q. Tang, C.-b. Gong, M.H.-W. Lam, Review of the recent progress in photoresponsive molecularly imprinted polymers containing azobenzene chromophores, Anal. Chim. Acta 900 (2015) 10-20.

[21] W. Chen, Y. Ma, J. Pan, Z. Meng, G. Pan, B. Sellergren, Molecularly imprinted polymers with stimuli-responsive affinity: progress and perspectives, Polymers 7 (2015) 1689-1715.

[22] A.J. East, N.J. Madison, Anisotropic Wholly Aromatic Polyester Derived from 4 hydroxy-4'-carboxy Azobenzene and Process for Preparation, US Patent, 1981. No 4285852 A. 
[23] H.J. Haitjema, R. Buruma, G.O.R. Alberda van Ekenstein, Y.Y. Tan, G. Challa, New photoresponsive (meth)acrylate (co)polymers containing azobenzene pendant sidegroups with carboxylic and dimethylamino substituents-I. Synthesis and characterization of the monomers, Eur. Polym. J. 32 (1996) 1437-1445, https://doi.org/10.1016/S0014-3057(96)00076-6.

[24] C. Gong, M.H.-W. Lam, H. Yu, The fabrication of a photoresponsive molecularly imprinted polymer for the photoregulated uptake and release of caffeine, Adv. Funct. Mater. 16 (2006) 1759-1767, https://doi.org/10.1002/ adfm.200500907.

[25] S. Grimme, Semiempirical GGA-type density functional constructed with a long-range dispersion correction, J. Comput. Chem. 27 (2006) 1787-1799, https://doi.org/10.1002/jcc.20495.
[26] M.W. Schmidt, K.K. Baldridge, J.A. Boatz, S.T. Elbert, M.S. Gordon, J.H. Jensen, S. Koseki, N. Matsunaga, K.A. Nguyen, S.J. Su, T.L. Windus, M. Dupuis, J.A. Montgomery, General atomic and molecular electronic structure system, J. Comput. Chem. 14 (1993) 1347-1363, https://doi.org/10.1002/ jcc.540141112.

[27] T. Yanai, D.P. Tew, N.C. Handy, A new hybrid exchange-correlation functional using the Coulomb-attenuating method (CAM-B3LYP), Chem. Phys. Lett. 393 (2004) 51-57, https://doi.org/10.1016/j.cplett.2004.06.011.

[28] A.V. Marenich, C.J. Cramer, D.G. Truhlar, Universal solvation model based on solute electron density and on a continuum model of the solvent defined by the bulk dielectric constant and atomic surface tensions, J. Phys. Chem. B 113 (2009) 6378-6396, https://doi.org/10.1021/jp810292n. 